\title{
Connectivity enhancement due to film flow in porous media
}

\author{
Marcel Moura $\odot,{ }^{*}$ Eirik Grude Flekkøy, and Knut Jørgen Måløy \\ PoreLab, The NJORD Centre, Department of Physics, University of Oslo, Norway \\ Gerhard Schäfer \\ Université de Strasbourg, CNRS, LHyGeS UMR 7517, F-67000 Strasbourg, France \\ Renaud Toussaint \\ Université de Strasbourg, CNRS, IPGS UMR 7516, F-67000 Strasbourg, France \\ and PoreLab, The NJORD Centre, Department of Physics, University of Oslo, Norway
}

(Received 22 January 2019; published 24 September 2019)

\begin{abstract}
We study the effects of connectivity enhancement due to film flow phenomena on the drainage of an artificial porous medium and the relative influence of gravity for such effects. The medium is initially fully saturated with a liquid (wetting phase), which is displaced by air (nonwetting phase). Our setup allows us to directly visualize the dynamics of the flow and, in particular, to pinpoint which pore invasion events are due to film flow phenomena. We have observed the formation of an active zone behind the liquid-air interface, inside which film flow drainage events are more likely to occur. Understanding the basic mechanisms of film flow in artificial porous media is of relevance for the analysis of real three-dimensional porous systems, in which gravity cannot be neglected and film flow is bound to be present.
\end{abstract}

DOI: 10.1103/PhysRevFluids.4.094102

\section{INTRODUCTION}

The study of the basic mechanisms behind fluid flow in porous media has received considerable attention from the scientific community in the past decades [1-10]. The theme is of importance not only because of its inherent physical interest (pattern formation [11-17], intermittent dynamics [2,18-24], collective phenomena [25-29], etc.) but also because of direct environmental applications and economical aspects. Hydrologists, for example, have been interested in the description of flows inside soils and rocks, in the study of groundwater flows [30], and in the treatment of soil contaminants [31]. The dispersion of contaminants in porous and fractured networks [32] and the formation and evolution of fractures [33] have also been subjects of recent studies. On the industrial side it is important to consider that basic concepts of flow in porous media lie in the core of many of the techniques employed, for example, in the prospection and exploration of oil and gas [34-36], in the capture and storage of carbon dioxide [37] and in the development of modern multifunctional porous materials for solar cells [38] and other related energy applications.

The theoretical and experimental studies of flow in porous media have been closely followed by the development of several numerical schemes tailored to reproduce the fluid dynamics under specific flow conditions. For example, very slow drainage flows in the so-called capillary regime (in which capillary forces dominate the dynamics) are commonly described by the invasion percolation (IP) algorithm [39] while the diffusion-limited aggregation algorithm (DLA) [40-42] captures the

*marcel.moura@fys.uio.no 
morphology and dynamics of faster drainage flows (dominated by viscous forces). The development and application of such growth models for porous media flows is motivated, among other reasons, by the difficulty in fully integrating the Navier-Stokes equations [43] inside porous networks. The complicated geometry of pores and throats in the medium renders the problem too demanding from the numerical point of view and analytical solutions are only available for very simple geometries [44]. In order to develop a reasonable growth model, one has to understand the basic mechanisms behind the pore-scale dynamics [26], including effects from the contact line dynamics [45], and how those mechanisms interact to yield the macroscopic properties of the flow. Understanding the relative importance of nontrivial flow mechanisms such as film flow is important to allow a precise judgment on whether or not a given flow condition can be appropriately described by a growth model such as IP or DLA (both models, in their standard formulations, do not incorporate film flow phenomena). Previous numerical and experimental studies have shown the importance of effects derived from film flow phenomena in a series of systems, such as the gravity-driven infiltration of a porous network [46], drainage and imbibition in etched networks [47], film flow along fractured rocks [48], transport in a network of capillary tubes, and drying of oak wood [49]. These studies are joined by a growing body of recent experimental works which endorse the idea that three-dimensional (3D) effects associated to the formation and interconnection of thin liquid films are crucially important to the morphology and dynamics of porous media flows, even in quasi-two-dimensional (quasi-2D) systems [50]. An important step in the modeling of two-phase flows was given recently in Ref. [51] in which the incorporation of wettability and film flow effects into a model similar to invasion percolation has led to the appropriate description of quasi-2D displacements in porous media, particularly in the challenging strong imbibition regime.

In this paper we will use the term film flow to describe a type of fluid transport that does not happen via the usual pathways through the bulk of pores and pore throats. More specifically, we define film flow as fluid flow along a particular type of fluid structure. This structure is characterized by two features: it must connect (directly or indirectly) to the outlet of the system, so that it may act as a drainage pathway, and it cannot fully span the channels where flow takes place. The latter criterion means that in the immediate vicinity of any region where film flow takes place, both fluid phases must be present. We incorporate in our definition the effects of corner flow (as those happening particularly in angular porous media [52-54]), flow via thin liquid films, and flow through capillary bridges, the latter being of particular importance to the results presented here.

\section{DESCRIPTION OF THE SETUP AND THE EXPERIMENT'S DYNAMICS}

\section{A. Experimental setup}

We make use of a synthetic transparent quasi-2D porous network to analyze the flows. The natural difficulties related to the visualization of flows in natural porous media, such as rocks and soils, has led many authors to employ artificial samples in their studies $[9,22,41,47,55,56]$. Figure 1 shows a diagram of the experimental setup used here. The medium is composed of a modified Hele-Shaw cell [57], filled with a monolayer of glass beads with diameters $a$ in the range $1.0<a<1.2 \mathrm{~mm}$. Those beads are randomly thrown onto the sticky side of a contact paper, whose nonsticky side is then fastened to a rigid rectangular Plexiglas plate. The voids in between the beads form the porous network. Silicon is used to define the in-plane boundaries of the porous medium and a second contact paper sheet is glued onto the beads (once again with the sticky side facing the beads). This whole structure is placed on top of a water pressure-cushion. This cushion is formed by a membrane filled with water and connected to a reservoir placed at a fixed height of $\sim 3 \mathrm{~m}$. Its purpose is to press the beads (sandwiched in between the contact paper layers) against the upper Plexiglas plate, thus assuring the quasi-2D structure of the medium and also guaranteeing that none of the beads will move during the flow (despite the differences in bead diameters). The dimensions of the porous networks are $34.5 \mathrm{~cm} \times 36.5 \mathrm{~cm}$, where the first number denotes the length of the network (measured in the inlet-outlet direction) and the second 


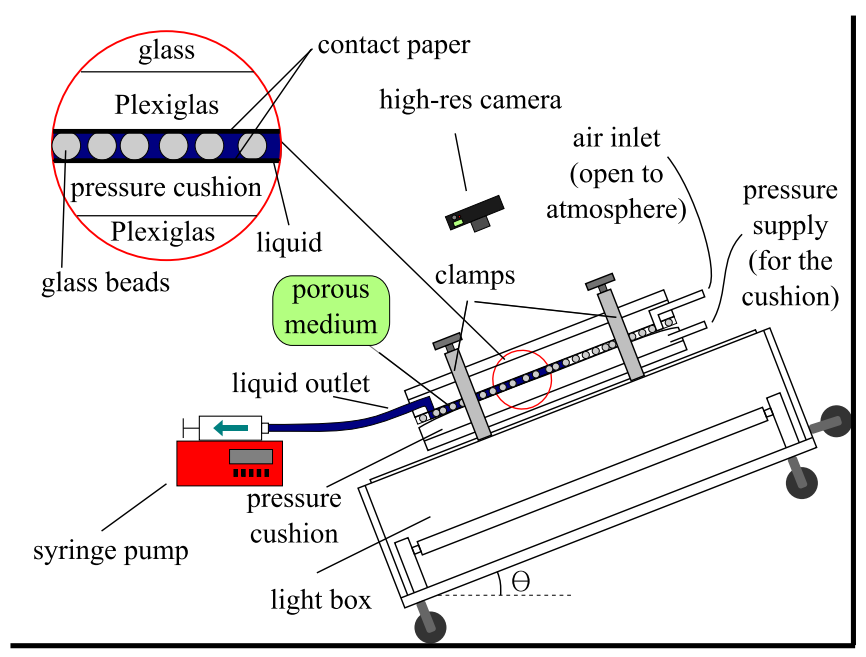

FIG. 1. Diagram of the experimental setup showing the cell with the porous medium made of a single layer of glass beads. The camera, light box, and cell are all connected to a common frame that can be inclined by an angle $\theta$, as shown. The liquid outlet on the left is connected to a syringe pump that withdraws the liquid. The water inlet for the cushion (on the right) is connected to a bottle which is kept at a high level $(\approx 3 \mathrm{~m})$. This is to keep the cushion pressurized. There is no connection between the water in this cushion and the fluids in the porous medium.

is its width (measured in the perpendicular direction). A heavy glass plate is placed on top of the model, to avoid possible issues with bending of the Plexiglas plate and to increase the overall robustness of the setup. Channels for the inlets and outlets were previously milled in the Plexiglas plate and cuts were made in the upper contact paper such that the liquid can be injected into and withdrawn from the medium. The system is placed on the top of a movable light box, which can be tilted in order to control the relative importance of gravitational effects on the dynamics (the component of the gravitational acceleration in the inlet-outlet direction is $g_{y}=g \sin (\theta)$, where $\theta$ is the inclination angle of the model with respect to the horizontal level (see Fig. 1). Rails on the wall guide the motion of the light box. From top to bottom the layers of the system are as follows: glass plate-Plexiglas plate-contact paper sheet-porous matrix (glass beads)-contact paper sheet-pressure cushion-Plexiglas plate. Images of the flow are taken from the top by a digital camera (NIKON D7100) fastened to a frame attached to the light box (in such a way that the relative position of the camera to the model is the same, irrespective of the inclination angle $\theta$ ). The porous matrix was initially filled with a wetting viscous liquid composed of a mixture of glycerol ( $80 \%$ in weight) and water (20\% in weight) whose kinematic viscosity, density, and surface tension (with respect to air) were measured to be, respectively, $v=4.25 \times 10^{-5} \mathrm{~m}^{2} / \mathrm{s}, \rho=1.205 \mathrm{~g} / \mathrm{cm}^{3}$, and $\gamma=0.064 \mathrm{~N} / \mathrm{m}$ (see Ref. [56] for additional details). All experiments were performed at a constant withdrawal rate of $q=0.6 \mathrm{ml} / \mathrm{h}$. The capillary number can be calculated as $\mathrm{Ca}=\rho v q / \Sigma \gamma=3.7 \times 10^{-7}$, where $\Sigma=3.65 \times 10^{-4} \mathrm{~m}^{2}$ is the cross section of the model. Since the medium is initially completely wet by the liquid, the contact angle (measured inside the wetting phase) is always found to assume low values, although its exact value varies during a pore invasion due to dynamical effects.

\section{B. Description of the dynamics}

In the drainage experiments performed, air (nonwetting phase) slowly displaces the liquid (wetting phase) previously filling the pores. The dynamics is driven by a syringe pump which withdraws liquid from the model's outlet (the air inlets on the opposite side of the model are open 
to the atmosphere). We have chosen to withdraw the liquid instead of pumping air in order to avoid possible compressibility effects in the air phase [58].

The regular pore-invasion mechanism during drainage is as follows: for the nonwetting phase to invade a given pore, the local value of the capillary pressure $p_{c}$, i.e., the pressure difference between the nonwetting and wetting phases, $p_{c}=p_{n w}-p_{w}$, must be high enough to overcome the capillary pressure threshold $p_{c}^{t}$ associated with the geometry of the pore throat giving access to that specific pore. Narrower pore throats present higher values of capillary pressure threshold in accordance with the Young-Laplace equation [59], and are, therefore, harder to invade. The pressure in the nonwetting phase (air) equals the atmospheric pressure and is taken to be constant during the experiment. In the presence of a gravitational field, the pressure in the wetting phase increases linearly in the inlet-outlet direction, due to a hydrostatic component, i.e., $p_{w}=p_{w}^{\text {out }}-$ $\rho g \sin (\theta)(L-y)$, where $p_{w}^{\text {out }}$ is the pressure in the wetting phase at the outlet of the model, $L$ is the model's length, and the distance $y$ is measured from the inlet of the model (see axis orientation in Fig. 8). As a consequence, the capillary pressure also becomes position dependent, being given by

$$
p_{c}=p_{c}^{\text {out }}+\rho g \sin (\theta)(L-y),
$$

where $p_{c}^{\text {out }}$ denotes the capillary pressure at the outlet. During the dynamics, the capillary pressure builds up until the invasion of the first pore for which the condition $p_{c}>p_{c}^{t}$ is verified. Notice that in the case of a horizontal system (where gravitational effects become negligible), the invasion would always be triggered in the widest pore throat available to the interface, since $p_{c}$ would not vary along that interface and the widest pore throat has the lowest capillary pressure threshold $p_{c}^{t}$, in accordance with the Young-Laplace equation [59]. When gravitational effects are present, since $p_{c}$ becomes position dependent, the invasion can happen through a pore throat that is not the widest one available, as long as it is the one for which the condition $p_{c}>p_{c}^{t}$ is first verified. Once the invasion of a pore happens, a new set of pore throats becomes available for the interface and if one (or more) of these throats happen to have sufficiently low values of $p_{c}^{t}$, they can also be immediately invaded, giving rise to the characteristic burst dynamics observed in such flows [1,2,5,22,60-62].

The liquid displaced in the pore invasion event leaves the system from the model's outlet and goes into the syringe in the pump. Notice that since the liquid is incompressible, if a portion of liquid gets completely surrounded by air-filled pores, it is in principle trapped inside the medium, since it cannot flow through the bulk of the air-filled pores. This effect has been successfully incorporated via a trapping rule in the invasion percolation algorithms used to study slow drainage flows in porous media $[39,61]$.

The mechanism described is responsible for the invasion of the majority of pores in slow drainage experiments. Nevertheless, sometimes it occurs that some trapped liquid clusters do indeed reduce in size with time and, in some cases, completely disappear, in spite of being surrounded by pores filled with the air phase. Figure 2 shows an example of this kind of invasion. In frame I (top right) we see an apparently trapped cluster of liquid. All pores surrounding it are filled with air, therefore, given the incompressibility of the liquid, one would expect that the liquid forming the cluster would remain trapped. Nevertheless, as can be seen in the figure, as time passes the cluster is slowly drained until almost disappearing in frame III after an interval of $\approx 10 \mathrm{~h}$. The explanation for the drainage of that cluster is film flow. Small portions of liquid are left behind as thin films around the glass beads after the main front has passed. Sometimes liquid films from adjacent glass beads are connected via a capillary bridge. A fortunate succession of such bridges can connect the supposedly trapped cluster to the bulk of the defending fluid, which is then connected to the outlet of the model. This is the pathway through which liquid in the cluster can be drained. In the inset in the left part of Fig. 2 we have marked with green arrows some of the capillary bridges that form a continuous connection (blue line) between that liquid cluster and another one in the bottom of the image (which could either be the defending liquid cluster connected to the outlet of the model or another intermediate liquid cluster). The formation and interconnection of capillary bridges thus introduces an additional flow pathway, which enhances the total connectivity of the medium and allows for the drainage of seemingly trapped fluid regions. 

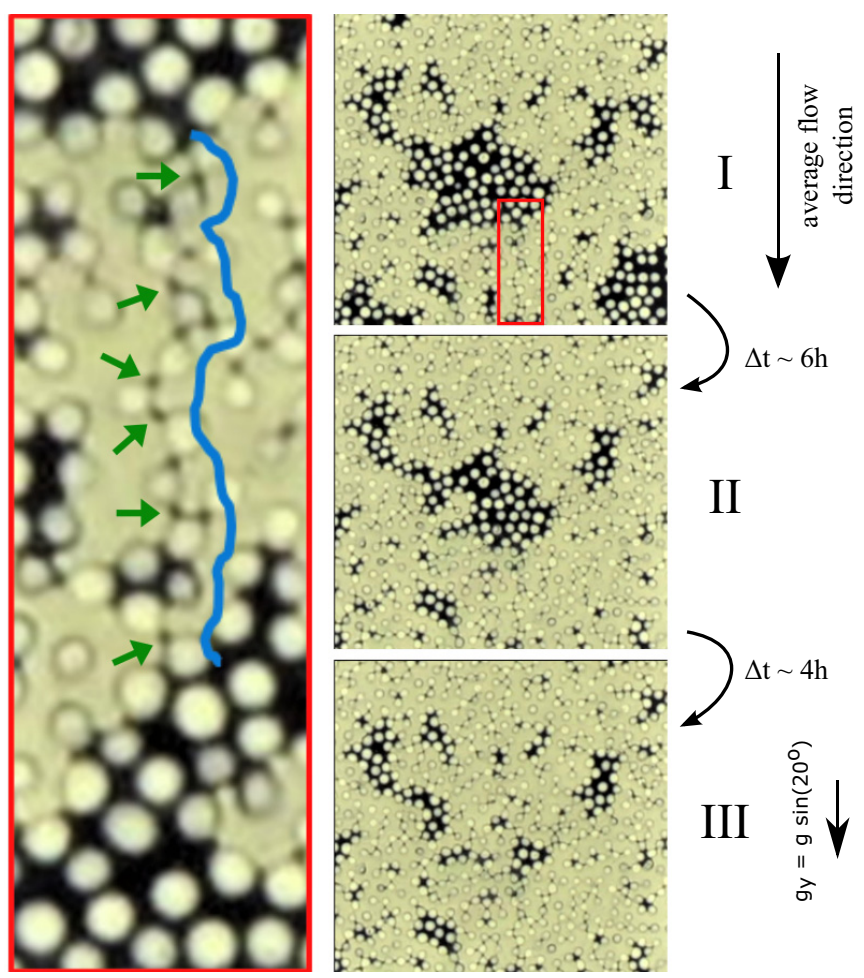

FIG. 2. Sequence of images showing the drainage of a supposedly trapped liquid cluster. The average flow direction is from top to bottom and the system is inclined by $20^{\circ}$, thus yielding a vertical component of the acceleration of gravity $g_{y}$ as shown. The time difference between frames I and III is $\approx 10 \mathrm{~h}$. The zoomed inset on the left (corresponding to the red rectangle in frame I) shows the path of interconnected capillary bridges through which film flow takes place, with some of the bridges marked by green arrows.

In Fig. 3 we show a macro image of a small segment of an experiment in which the capillary bridges can be seen more clearly (three of them are marked by arrows). The diameter of the glass beads is $\approx 1 \mathrm{~mm}$. The typical radius of one capillary bridge can be roughly estimated from this image as $r_{b}=0.15 \mathrm{~mm}$. Notice that this radius is certainly not necessarily the same for all capillary

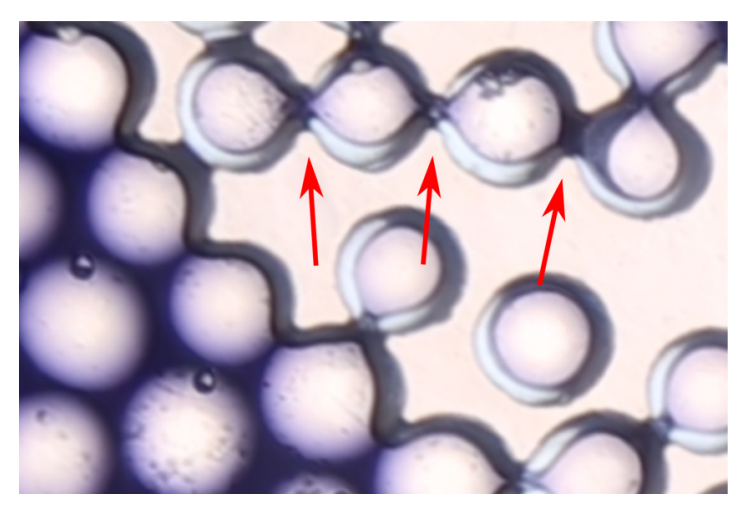

FIG. 3. Macro image of an experiment showing some capillary bridges (three of them are marked by arrows). The diameter of the glass beads is $\approx 1 \mathrm{~mm}$. 
bridges, depending on a number of parameters and most notably on the pore throat size (distance between glass beads), larger throat sizes typically yielding narrower capillary bridges. If a throat size is too large, a bridge in it would be unstable so it is expected that very large pore throats do not hold capillary bridges, as seen in Fig. 3. This topic will be further discussed in Sec. III B.

The interconnection between pores via capillary bridges or similar film flow mechanisms depends on several factors, particularly on the history of the flow, i.e., on the order at which the pores have been initially invaded by air (via the regular invasion mechanism described previously), on specific details of the porous media structure [52-54], and also on the role played by gravity. In the case in which the density of the wetting phase is higher than that of the nonwetting phase (like in the experiments reported here), gravity plays a stabilizing effect over the front $[5,62,63]$, with direct consequences to the history of the sequential pore invasions, thus influencing the formation and orientation of capillary bridges in the model.

\section{Capillary bridges tracking algorithm}

We have devised an image analysis algorithm for tracking the capillary bridges in the system. A description of the algorithm follows: initially, the centers of all the glass beads in the model are identified. Then, we produce a Delaunay triangulation [64] to identify neighboring beads. Next, we identify all the midpoints along the lines connecting the centers of neighboring beads (the midpoints of the lines forming the Delaunay triangulation). These midpoints are seen as the centers of the pore throats in the medium. We then consider a small line (with length of the order of the bead diameter) passing through those midpoints, in the direction perpendicular to the direction of the line connecting consecutive beads centers. This line is a perpendicular bisector of the line segment connecting the center of two neighboring beads. Finally, we examine the color intensity level along this perpendicular bisector, which is then used to classify the pore throats according to their contents into four categories as shown in Fig. 4. The color intensity can be measured according to different scales; for instance, one could convert the images to gray scale and then measure the gray level. In our case we have obtained better contrast by considering only the red channel ( $R$ in the RGB) of the images and then measuring the color intensity in that channel. If the color intensity is approximately constant along the bisector, it means that the pore throat belongs to the bulk of one of the phases (air if the color intensity value is higher than a certain threshold or liquid if this value is lower than this threshold). If the color intensity transitions from low to high or vice versa, it means that this pore throat belongs to the edge of a liquid cluster. Finally, if the color intensity goes from high to low to high, it means that the center of the pore throat contains a thin layer of liquid, while the pore bodies on both sides of the pore throat contain air. This is a capillary bridge and this is the pattern that we are interested in tracking here (see Fig. 4).

The tracking algorithm previously described was employed in the experiments performed and an example of the resulting capillary bridges tracking can be seen in Fig. 6. It is important to notice that the algorithm described is only one among many possible solutions to the problem. When higher-resolution images are available, other solutions may become more interesting/efficient.

\section{Identification of film flow drainage events}

In order to study the effects of film flow in our experiments, we have developed an image analysis algorithm to pinpoint, among the set of all invaded pores, those invaded due to film flow. The algorithm makes use of an adaptive masking routine to perform the task. A description follows: for a given time step, the current picture of the invasion is binarized to identify the instantaneous invasion region [see Fig. 5(a)]. The invading front (main liquid-air interface) is then computed, as shown by the red line in Fig. 5(b) [the region corresponding to the square in (a)]. A following snapshot of the invasion ( $\sim 2 \mathrm{~h}$ later) is superposed to this image and the difference between the two images marks the new pores that were invaded in this interval [cyan region in Fig. 5(b)]. Most of those pores lie in the defending cluster (below the front), but a small portion of them lie in the region 


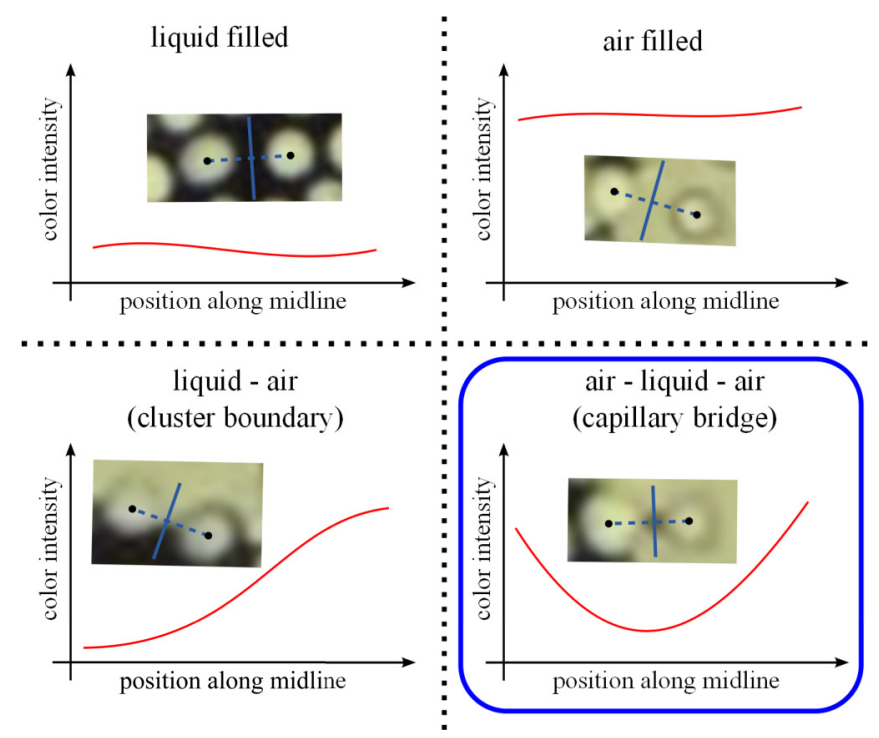

FIG. 4. Diagram showing the algorithm employed to track the capillary bridges. For each pore throat we compute the color intensity plot along a perpendicular bisector segment (solid blue lines), a line passing through the center of the pore throat along a direction perpendicular to the direction connecting the center of the beads (dashed blue lines). There are four possible outcomes for this plot. If the pore throat lies in the bulk of one of the phases, the color intensity is approximately constant, giving a higher value for the air-filled pores (lighter color) and a lower value for the liquid-filled pores (darker color), as shown in the two diagrams on the top. If the pore throat belongs to a cluster boundary, it is partially liquid and partially air filled, and the plot shows a transition from lower to higher values or vice versa (bottom left). When a capillary bridge is present, the color intensity goes from high to low to high, indicating the thin liquid bridge in between air-filled pore bodies (bottom right). It is this kind of pattern that we track for all pore throats in the model in order to pinpoint the position of the capillary bridges.
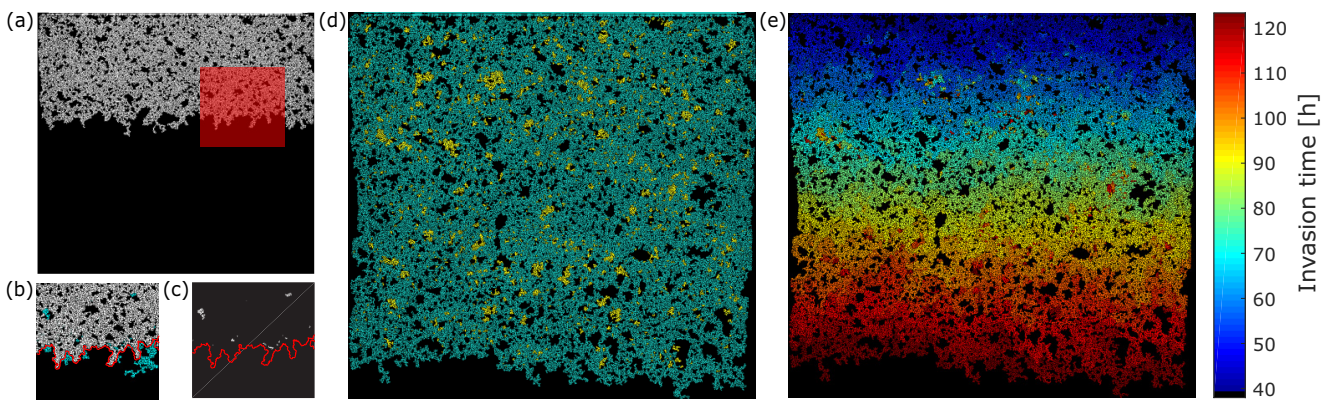

FIG. 5. Identification of film flow events. (a) A typical snapshot of the experiment with an inclination of $20^{\circ}$, after thresholding to isolate the invading air phase (white). (b) Detail from (a) (red square) showing a differential image between two snapshots separated by $\sim 2 \mathrm{~h}$. The area in cyan denotes pores that have been invaded in this interval. The majority of the invasion happens along the front (red line), but some events occur behind the front [film flow events, isolated in (c)]. In (d) we show the invasion map at breakthrough. The green area shows pores that were invaded via the usual mechanisms while the yellow area denotes those pores invaded via film flow effects. In this experiment, film flow effects were responsible for an increase in saturation of $8.3 \%$. A full spatiotemporal map of the invasion is shown in (e), where the color code denotes the invasion time (in hours) of a given pore. 


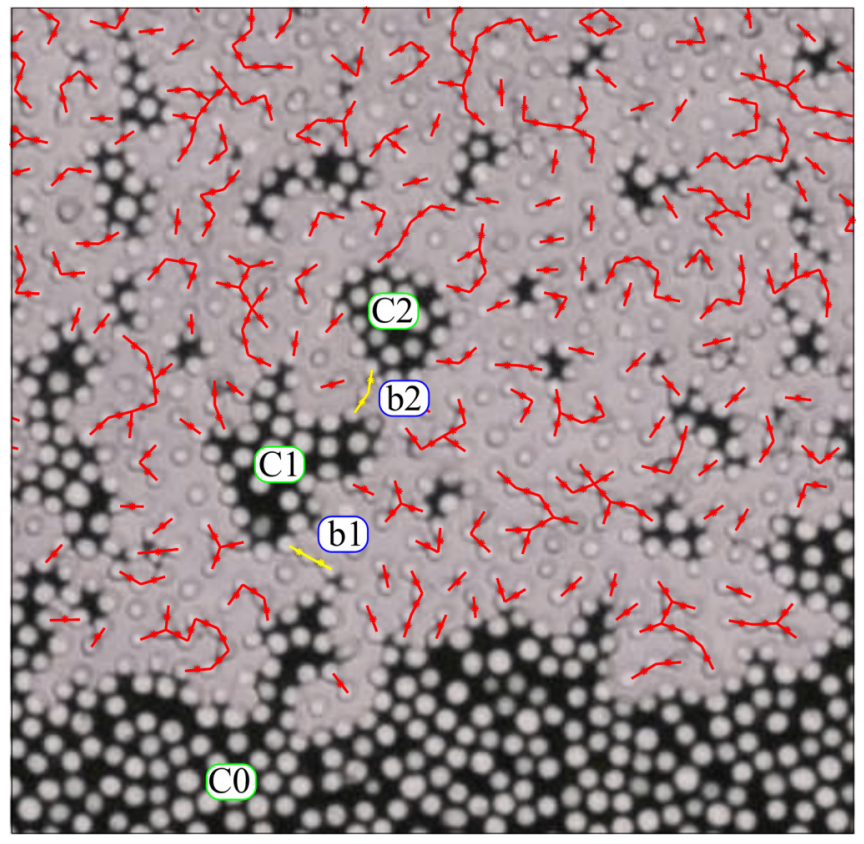

FIG. 6. Capillary bridges tracking. The red lines connect the centers of beads through which a continuous capillary bridge connection exists. The bridges positions are marked by an asterisk on the lines. The liquid in the cluster $\mathrm{C} 1$ can eventually be withdrawn to the defending cluster $\mathrm{C} 0$ through the capillary bridges pathway b1, marked in yellow. Similarly, the liquid in cluster C2 can flow into the cluster C1 through the capillary bridges in b2, also marked in yellow. From there, it could also flow into the defending cluster C0 through b1. This interconnection mechanism, through bridges and intermediate clusters, effectively enhances the overall connectivity of the liquid phase.

above the invasion front and are disconnected from it. Those pores, not being directly connected to the defending cluster, are the ones whose invasion happens via film flow effects. Figure 5(c) shows an example of the resulting tracking in this small section and in Fig. 5(d) we show the film flow tracking applied to the whole model until breakthrough. The pores marked in green are the ones invaded via the usual drainage mechanism, while the ones in yellow were invaded via film flow. In Fig. 5(e) we show a full spatiotemporal map of the invasion of all pores. The colormap assigns the time in hours for the invasion of a given pore. Notice that, comparing the yellow islands in Fig. 5(d) with the spatiotemporal map in Fig. 5(e), some of the film flow drainage events happened much later than the time in which the main invasion front first passed by.

\section{RESULTS AND DISCUSSIONS}

\section{A. Connectivity enhancement via capillary bridges}

The formation of capillary bridges is not a sporadic event and, depending on the properties of the fluids involved and on the morphology of the porous medium, transport through these bridges can play a crucial role in the overall flow behavior. We have employed the capillary bridges tracking algorithm presented in Sec. II C to directly visualize the enhanced connectivity introduced by the capillary bridges in the porous medium. Figure 6 shows the result of such tracking applied to a portion of the medium. We have drawn red lines connecting the centers of beads which happen to be linked through a capillary bridge (marked with a small red star in the middle of each line segment). We denote $\mathrm{C} 0$ the defending liquid cluster, connected to the outlet of the model from 
which the liquid is withdrawn. Although being apparently trapped by the air phase, the cluster $\mathrm{C} 1$ is still connected to $\mathrm{C} 0$ (and thus to the outlet of the system) via the capillary bridges pathway denoted b1 (in yellow in the figure). Therefore, the liquid in $\mathrm{C} 1$ can still be withdrawn. Similarly, the cluster $\mathrm{C} 2$ can be withdrawn through the pathway $\mathrm{b} 2$ into $\mathrm{C} 1$ and then through $\mathrm{b} 1$ into $\mathrm{C} 0$. The kind of mechanism just described introduces the possibility of drainage of pores that lie far away from the boundary of the defending cluster. The typical distance for this film flow drainage to occur depends on the probability of having a continuous pathway of bridges and liquid-filled pores connecting the particular pore in question to the boundary of the defending cluster $\mathrm{C} 0$. This distance sets in a characteristic length scale for the film flow dynamics, and is a function of the pore geometry, the rheology, and wetting properties of the fluids involved and of possible capillary bridge snapping-off mechanisms (which can potentially be influenced by gravitational effects).

By considering the fluid transport via such capillary bridges, we are effectively introducing an additional secondary network, where the primary network is understood as the usual set of interconnected pores and pore throats. The typical timescale for transport in this secondary network is expected to be longer than in the primary one, due to the fact that the average widths of the capillary bridges and other film flow sources are smaller than the average pore size. In the usual terminology of porous media flows, we would say that we expect the secondary network to have a lower effective permeability than the primary one (here the term permeability is used only figuratively to address the resistance to the flow). Nevertheless, for long-lasting processes in the capillary regime, transport through the secondary network can lead to very significant changes in the phases saturation and overall displacement structures, as we will see later on.

An additional important distinction between the two networks described is that while the primary network is mostly static (for nondeformable consolidated porous media), the secondary one is dynamic, depending strongly on the flow conditions and fluids involved. The formation and snapping off of capillary bridges can drastically interfere with the connectivity properties of the secondary network, thus introducing a new source of complexity for slow drainage flows (discussed later on in Sec. III F). Further on we will show that one important consequence of the enhanced fluid connectivity through the secondary network is the formation of a film flow active zone behind the main liquid-air interface where drainage due to film flow has a higher probability of occurrence.

\section{B. Capillary bridges size distribution and stability}

As previously mentioned, the occurrence of such capillary bridges is certainly not rare. In order to give quantitative validation to this statement, we plot in Fig. 7 two histograms showing the distribution of capillary bridges of a given size $d$ and the total distribution of pore throats of size $d$. The size is understood as the distance between the neighboring glass beads that limit the capillary bridge or the pore throat. The data was computed using the final image of the experiment performed at $20^{\circ}$ inclination, but we would not expect major qualitative differences had the measurement been performed at an earlier stage. (As we will see later in Sec. III E, the total number of capillary bridges inside a given subwindow in the model does not seem to vary too much as the subwindow's position is changed. As long as the number of bridges is large enough to overcome fluctuations due to poor statistics and/or boundary effects, it is reasonable to expect that the capillary bridge size distribution does not change much either.) The analysis of Fig. 7 shows that from the total number of pore throats in the model, approximately $15 \%$ were filled with capillary bridges. One can also notice that the capillary bridges are not homogeneously distributed over the pore throats, meaning that some throat sizes are more prone to host bridges. This can be more easily seen by computing the ratio between the blue and brown histograms, which gives an estimate of the conditional probability that a given pore-throat size in the model ends up hosting a capillary bridge. The result is shown in the inset of Fig. 7. We see that for very small pore throats (say smaller than $0.3 \mathrm{~mm}$ ) the probability of hosting a capillary bridge grows to more than $40 \%$, a significant increase in comparison with the $15 \%$ probability found when considering all pore-throat sizes. 


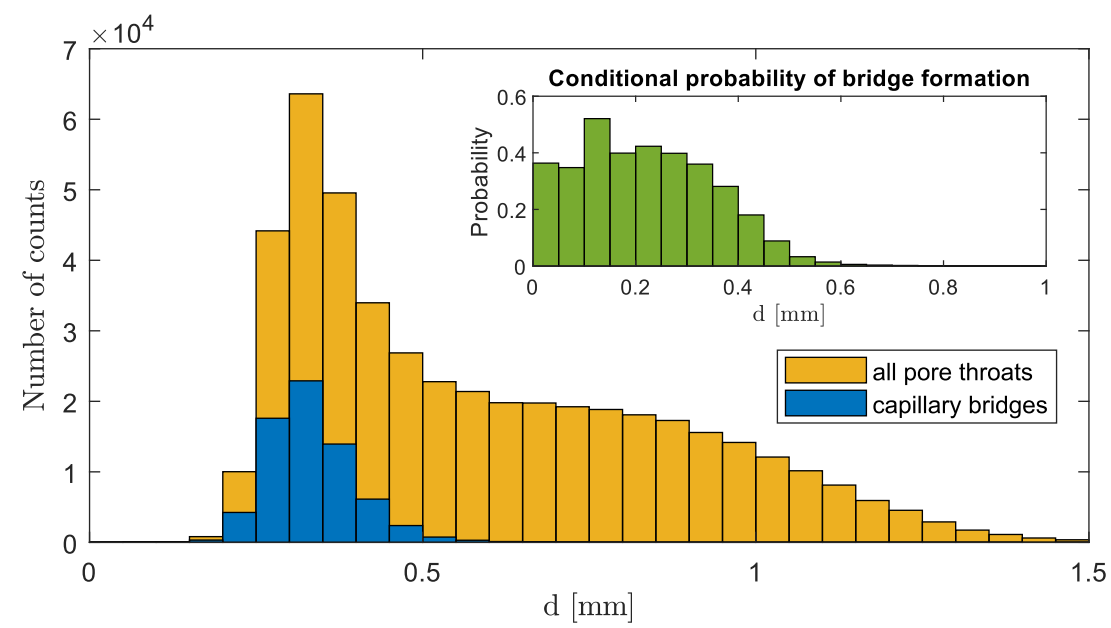

FIG. 7. Histogram showing the total frequency of pore throats (brown) and capillary bridges (blue) in an experiment with $20^{\circ}$ inclination. In the inset it is shown an estimate of the conditional probability that a pore throat of a given size will host a capillary bridge (ratio of the histograms in the main figure). Notice that if a pore throat is wider than a given threshold $(\approx 0.5 \mathrm{~mm}$ in this case) the formation of a capillary bridge in that throat is very unlikely.

We can also see that it is extremely unlikely that a capillary bridge is formed in pore throats larger than a certain threshold (of about $0.5 \mathrm{~mm}$ in our systems). The reason for the existence of a maximum pore-throat length capable of hosting capillary bridges can be intuitively understood based on two main effects: hydrodynamic instability and the nature of the drainage process. When a cylindrical film of liquid is stretched beyond a certain length, it tends to break up as a result of the Plateau-Rayleigh instability [65] (a classic example being the breaking of a thin stream of water from a tap into a sequence of droplets). Following Rayleigh [65] we see that a cylindrical film is unstable for all perturbations having wavelength $\lambda$ larger than the circumference $2 \pi r_{b}$ of the film, i.e., the perturbation is unstable if

$$
\lambda>2 \pi r_{b} .
$$

If the capillary film has length $d$, the boundary condition that the perturbation must vanish at the extremes of the bridge yields

$$
\lambda=\frac{2 d}{n},
$$

where $n$ is a positive integer. The largest wavelength is then $\lambda=2 d$ and substituting this in Eq. (2) we see that the cylindrical film is unstable if its length exceeds the critical length

$$
d_{c}=\pi r_{b} .
$$

Considering the capillary bridges as cylindrical films to a first-order approximation and using the estimate of $r_{b}=0.15 \mathrm{~mm}$ (see Fig. 3) we get $d_{c}=0.47 \mathrm{~mm}$, which is in accordance with the fact that no bridges are formed for pore throats wider than $d=0.5 \mathrm{~mm}$ (see the inset of Fig. 7).

Although the simple calculation above provided a good estimation for the maximum pore-throat size that can host a capillary bridge, hydrodynamic instability is certainly not the only factor in play here. As previously mentioned, due to the nature of the drainage process, larger pore throats are typically invaded more easily by the liquid-air interface. Since the standard invasion happens through the bulk of the pore throat, it leaves no chance for the formation of capillary bridges in those larger pore throats. 


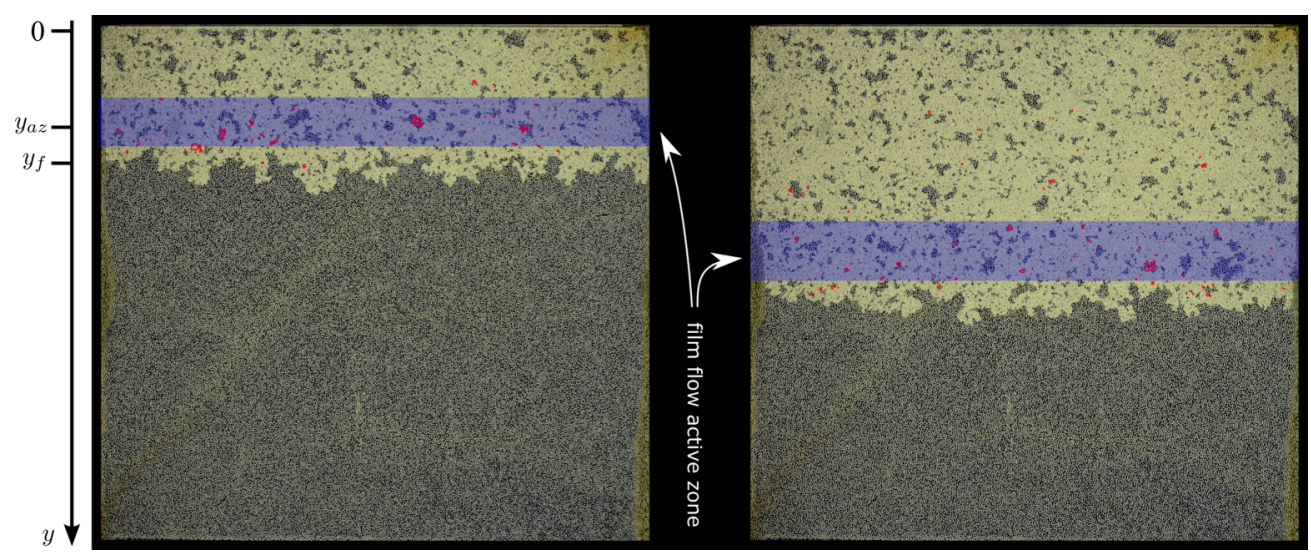

FIG. 8. Drainage of a model with an inclination of $20^{\circ}$. The average flow direction and gravity component are from top to bottom, along the $y$ axis. In red we mark the pores that were invaded due to film flow phenomena between the instant of the picture and another image taken $\approx 2 \mathrm{~h}$ earlier. The time difference between the first and second frames is $\approx 24 \mathrm{~h}$. A film flow active zone (shown schematically as the blue bands in the figure) follows the liquid-air interface. The vertical positions $y_{a z}$ of the active zone and $y_{f}$ of the invading front are shown schematically.

\section{Impact of film flow drainage on the residual saturation}

As previously mentioned, film flow effects can lead to the drainage of liquid clusters that would otherwise be considered completely trapped. As a result of that extra drainage, the residual (irreducible) saturation of the wetting phase in the model can be considerably affected by film flow effects. Indeed, as shown in Fig. 5(d), the overall effect of film flow drainage has led to an increase in air saturation of about $8.3 \%$ for the experiment performed at $20^{\circ}$, with a corresponding decrease of the liquid saturation. This effect is far from negligible and becomes particularly relevant when one considers that several models used to describe porous media flows rely on constitutive relations (e.g., for the water retention and relative permeability curves) which are a function of the phase saturation. For example, the generalized Darcy law [66] used to describe the momentum equations in a representative elementary volume of a porous medium needs constitutive relationships in two-phase systems such as relative permeabilities of each fluid phase, which are themselves a function of phase saturation.

\section{The film flow active zone}

We have observed the existence of a film flow active zone, a region in which the drainage of pores due to film flow is more likely to occur. This region (henceforth termed simply the active zone) is concentrated behind the main invasion front, i.e., behind the interface between the invading air phase and the defending liquid cluster connected to the outlet. Figure 8 shows two frames from an experiment made with a $20^{\circ}$ inclination. The time interval between the frames is $\approx 24 \mathrm{~h}$. We have marked in red the contours of pores invaded due to film flow between one frame and a previous one $\approx 2 \mathrm{~h}$ earlier. As can be seen, the film flow drainage events happen more frequently in the vicinity of the invasion front. Film flow invasion far from this zone can also happen, but is much less likely.

In order to quantify this observation, we have measured the temporal development of the invading front position and its width (respectively, $y_{f}$ and $w_{f}$ ) and those of the film flow active zone (respectively, $y_{a z}$ and $w_{a z}$ ). This measurement is produced by obtaining the distribution of pixels belonging either to the front or to film flow drainage events and then considering its mean (defining the front and active zone positions, $y_{f}$ and $y_{a z}$ ) and the standard deviation with respect to this mean (defining the front and active zone widths, $w_{f}$ and $w_{a z}$ ). Notice that the widths of the front and active zone are here measured along the $y$ direction, while previously we had used the word width 


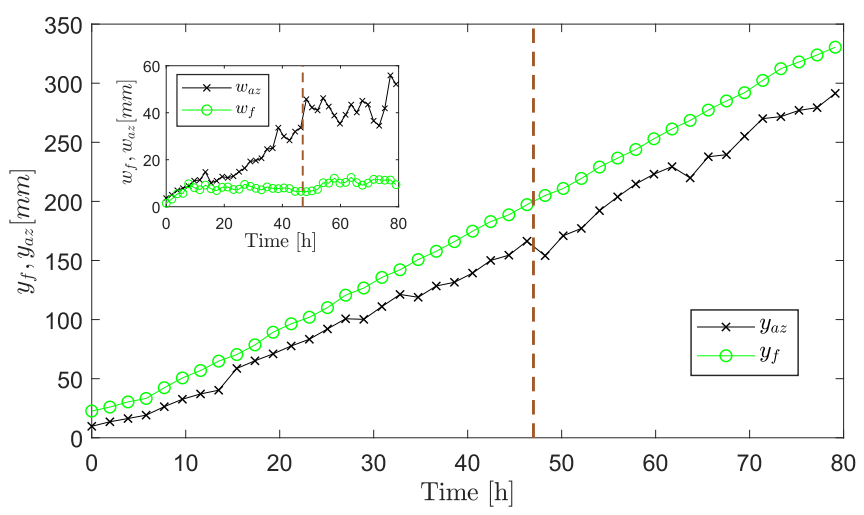

FIG. 9. Temporal development of the film flow active zone and the main liquid-air interface (the invading front) for an experiment with a $20^{\circ}$ inclination. In the main plot we show the average vertical position of the invading front $\left(y_{f}\right.$, green circles) and the film flow active zone ( $y_{a z}$, black crosses). After a transient time (brown vertical dashed line), the film flow active zone follows the invading front, moving at the same speed. In the inset we show the development of the widths of the invading front $\left(w_{f}\right.$, green circles) and of the film flow active zone ( $w_{a z}$, black crosses). While the invading front maintains a roughly constant width throughout the experiment, the width of the active zone develops for a much longer time.

to characterize the extension of the porous network itself along the perpendicular direction. We have considered all growth zones due to film flow occurring in a given time frame (chosen to be of $\approx 2 \mathrm{~h}$ ) and repeated the measurement to span the time range between the beginning of the invasion and the breakthrough, i.e., the instant at which the air phase first percolates, reaching the outlet of the model. Figure 9 shows the resulting measurements for the experiment shown in Fig. 8 with a $20^{\circ}$ inclination angle. The vertical position of the active zone ( $y_{a z}$, black crosses) closely follows the liquid-air interface $\left(y_{f}\right.$, green circles). This position is measured using a reference frame like the one shown in Fig. 8, with the origin at the inlet channel. After a transitional period (marked by the vertical brown dashed line), the active zone propagates with the same speed as the invading front, thus keeping a constant distance from it. The inset shows the evolution of the width of the invading front $\left(w_{f}\right.$, green circles) and of the active zone ( $w_{a z}$, black crosses). Notice that while the width of the invading front is essentially constant (it reaches a stationary regime much faster), the width of the active zone continues to grow for a much longer timescale. This growth stabilizes at around $t=47 \mathrm{~h}$ (vertical line), remaining roughly constant after that. The time needed to stabilize the width of the active zone corresponds to the transitional period observed in the main plot.

Special attention should be put on the observation just described that these two processes, the growth and stabilization of the invading front and that of the active zone, happen at very distinct timescales. The explanation lies in the fact that these two processes depend essentially on flow phenomena happening in two different networks: the drainage of pores forming the invading front happens through the usual network of pores and pore throats, the primary network, whereas the film flow drainage of pores behind the interface happens through the secondary network formed by the capillary bridges and/or other possible sources of corner and film flow, as shown in Fig. 6 and described in Sec. III A. The lower effective permeability of the secondary network is partially responsible for the longer timescale observed for the stabilization of the active zone width, but other factors are also important, such as the likelihood of capillary bridges formation and their subsequent snap off.

The active zone is more clearly observable for invasions happening at higher angles, and gets more dispersed as the angle is reduced. In order to see this quantitatively, we show in Fig. 10(a) a comparison of the active zone width $w_{a z}$ for four different angles: $0^{\circ}, 10^{\circ}, 20^{\circ}$, and $30^{\circ}$. From the analysis of this figure, one can see that the higher the gravitational component in the direction of the 
(a)

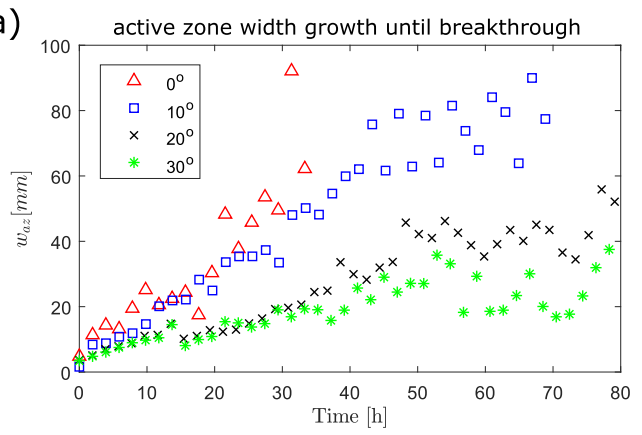

(b)

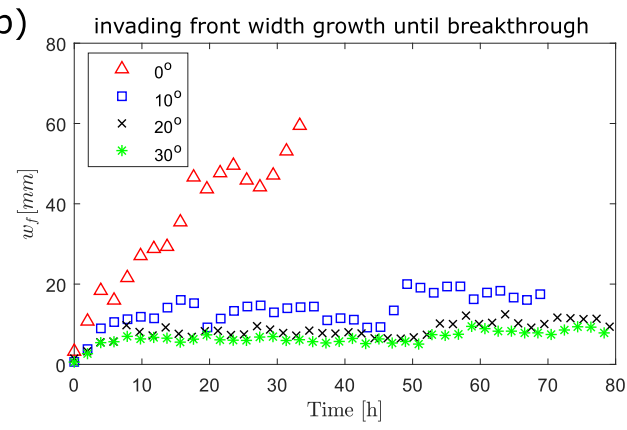

FIG. 10. Temporal evolution of the (a) active zone and (b) invading front width, as measured from its standard deviation with respect to the mean position. Measurements for $0^{\circ}, 10^{\circ}, 20^{\circ}$, and $30^{\circ}$. The higher the gravitational component (higher angles), the more compact the active zone and invading front are (smaller width). In the absence of the stabilizing effect from gravity, breakthrough happens at an early stage in the experiment with $0^{\circ}$, which is reflected in the sharp increase in the front width.

model (higher angles), the more compact and well defined (smaller width) the active zone is. For the flat model, breakthrough happens at an early stage and one cannot clearly observe a well-defined film flow active zone, which is reflected in the dispersion of the measurements in the figure for $0^{\circ}$. As this angle is increased, the front width moves towards a constant value as the flow develops. The larger the gravitational component, the smaller this value gets, thus suggesting that in systems in which gravity plays a major role, for example, in the case of the drainage of real 3D samples with a large density difference between the phases, film flow drainage becomes more and more relevant for the pores that lie in close vicinity to the interface between the phases. For completeness we also show the evolution of the width of the main invasion front in Fig. 10(b). As expected, for higher angles the invasion front becomes flatter (smaller width), due to the stabilization effect of gravity (the heavier liquid is in the bottom).

Although the data in Fig. 10(a) suggests a trend for the reduction in the width of the active zone as the angle increases, a better picture can be derived by computing the probability of film flow invasion events in the moving reference frame of the mean liquid-air interface (invading front). This is done in Fig. 11. This figure is computed as a histogram of the distances to the mean invading front of all

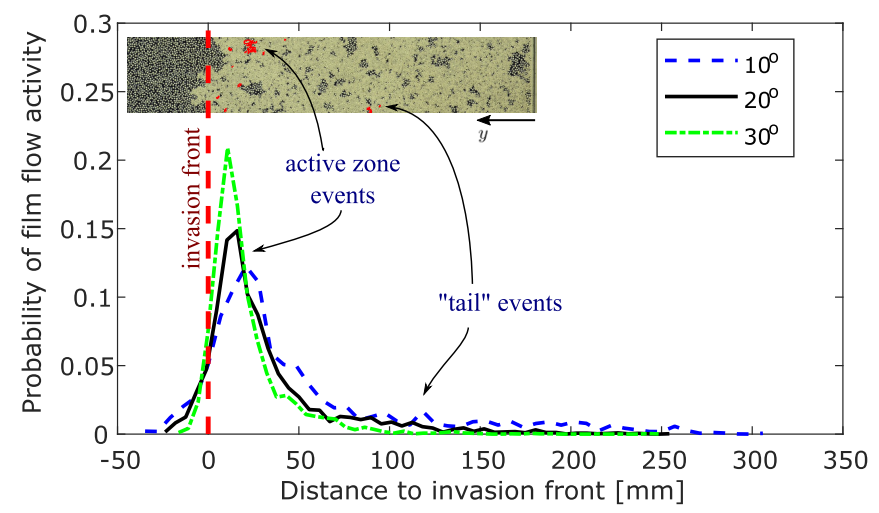

FIG. 11. Probability distribution of film flow events computed in the reference frame of the moving front. The measured data is shown for three inclination angles (see legend). Notice that apart from a few tail events, most of the film flow drainage occurs inside an active zone in the vicinity of the front. The position of the front is marked by the vertical dashed red line at the origin and the film flow active zone follows its motion. 
(a)

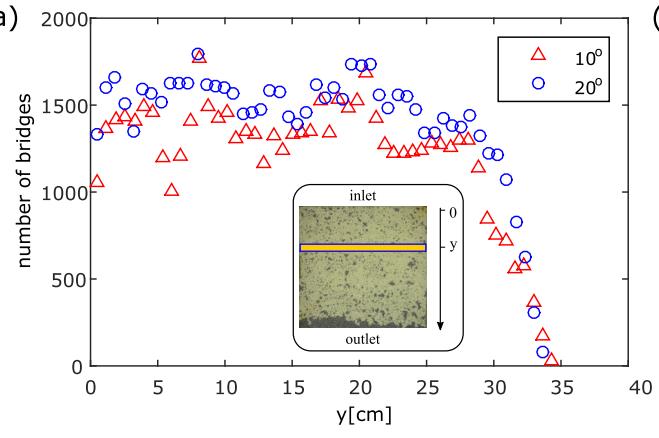

(b)

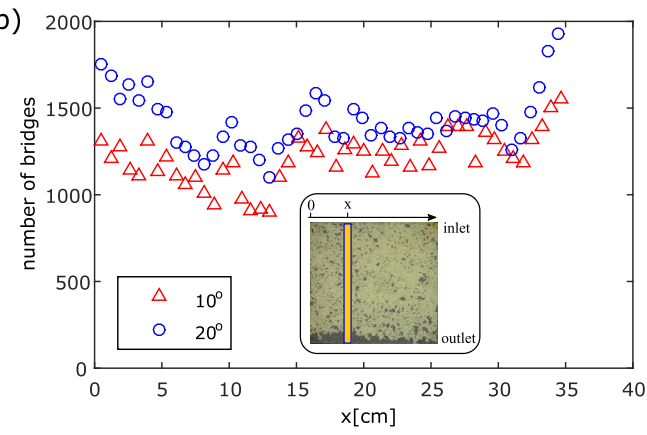

FIG. 12. Histogram showing the spatial distributions of capillary bridges. In (a) we show the essentially constant distribution inside subwindows in the inlet-outlet direction, as a function of the distance $y$ to the inlet. In the inset we show the orientation of the $y$ axis with respect to the model and a subwindow (made approximately three times taller than the ones used in the analysis, to aid visualization). In (b) we show the distribution in the perpendicular direction. Again, apart from fluctuations and boundary effects, the distribution is essentially constant.

pixels in the images that belong to pores invaded via film flow [yellow pixels in Fig. 5(d)]. Indeed we see that the film flow activity is more intense in an active zone that follows the motion of the front. As the angle is increased, the width of the active zone decreases and the zone seems to move closer to the invasion front (located at the origin, vertical dashed red line). The peaks of the film flow activity occur at the distances $21 \mathrm{~mm}$ for the experiment at $10^{\circ}, 16 \mathrm{~mm}$ for $20^{\circ}$, and $11 \mathrm{~mm}$ for $30^{\circ}$. Since the bead size in the models is $1 \mathrm{~mm}$, and that scale is similar to the pore scale, the distances reported in millimeters keep the same numerical values in pore-size units. In addition to the invasion in the active zone, we can also notice the existence of a few drainage events far from the front ("tail" invasion events). The statistics of these events is also of interest and could potentially give information about how likely (or unlikely) it is to have a very long capillary connection in the porous network (either through a long set of connected capillary bridges or other liquid films in the porous matrix).

Notice also that the data in Fig. 11 shows the occurrence of a few film flow events ahead of the mean position of the invading front (negative distances in the figure). This happens because the front is not completely flat. Even though gravity has a stabilizing (flattening) effect [62,63], the front is always rough and has a characteristic width [as previously seen in Fig. 10(b)]. Since this width is larger for smaller angles, the likelihood of film flow activity inside the front and ahead its mean position is also higher for smaller angles, as can be seen to the left of the vertical line in Fig. 11.

\section{E. Spatial distribution of capillary bridges}

We have employed the capillary bridges tracking algorithm outlined in Sec. IIC to measure the spatial distribution of capillary bridges. Figure 12(a) shows this distribution in the inlet-outlet direction for experiments tilted by $10^{\circ}$ and $20^{\circ}$. We have divided the inlet-outlet distance into 50 equal subwindows, each one spanning the whole porous medium width. The histogram shows the number of capillary bridges within each subwindow as a function of the distance $y$ between the subwindow's center and the inlet. This measurement is taken at the moment of breakthrough, i.e., when the air phase first reaches the outlet of the system. As expected, the number of bridges goes to 0 for subwindows close to the outlet, since that corresponds to a region mostly filled with the liquid phase and capillary bridges are only formed in the area invaded by air (we recall that a capillary bridge is formed by a thin layer of liquid in a pore throat for which the pore bodies on both sides of the throat have been filled with air; see Sec. II C). We can also observe that the overall number of capillary bridges in the system is larger for the experiment performed at a steeper angle. This is to be expected: since gravity plays a stabilizing effect on the front [Fig. 10(b)], the final air saturation 
increases as the angle increases. Since more pores are filled by air, there are more possibilities for the formation of capillary bridges in the steeper experiments (they cannot be formed in the bulk of the liquid phase).

For completeness we also measured the number of capillary bridges using subwindows moving in the perpendicular direction, i.e., along the width of the model. The result is shown in Fig. 12(b). We have noticed that close to both left and right boundaries the number of capillary bridges seems to increase. More studies are needed to fully understand this boundary effect, but it seems that the presence of the boundaries creates regions of higher film flow connectivity, an effect that may play an important role for applications in which transport properties are measured in the laboratory using bounded small core samples and the data is later extrapolated to larger scales (e.g., reservoir scale). Other capillary end effects associated to system boundaries have been previously reported in the literature in recent experiments [56] and simulations [67] and it is of pivotal importance to ensure that those effects do not contribute to spurious data in the measurements of the transport properties of a given sample. To avoid confusion we would like to state that the effects studied in Refs. [56,67] are mostly associated with the inlet and outlet boundaries, while the one suggested in Fig. 12(b) is associated with the side boundaries of the system.

\section{F. Capillary bridges snap off: Local versus global effects on the system's connectivity}

The snapping off of capillary bridges plays an important role in determining the connectivity properties of porous media in general, especially for slow flows happening on large timescales for which film flow effects are crucially important. In principle, for a given time, capillary bridges located closer to the inlet are on average older than those located far from the inlet, since their formation occurs when the air-liquid interface passes by a region, and this occurs earlier the closer we are from the inlet. Therefore, one would expect that for later times, the number of capillary bridges in the sample should decrease as one approaches the inlet, since the capillary bridges there were formed at an earlier stage of the flow (thus giving them more time to snap off). Following this line of reasoning, we would expect that at the end of an experiment, the number of capillary bridges close to the inlet should be significantly smaller than the number close to the outlet. We can test this idea by analyzing Fig. 12(a). Contrary to what we expected, in the timescale of our experiments, which is of $\sim 90 \mathrm{~h}$ between the beginning of the invasion and breakthrough, there did not seem be any clear drift in the total number of bridges. Apart from statistical fluctuations, the number of bridges along the model seems to be roughly constant (except from the region very close to the outlet where the number decays as previously explained due to the fact that that region is mostly filled by the liquid). This observation seems to indicate that the total number of snap-off events is not large enough to yield a major effect in the total capillary bridges number. Nevertheless it is worth mentioning that even a small number of snap-off events can lead to major changes in the secondary network transport properties, since the snap off of a single bridge could potentially disconnect large regions of the porous medium from the defending cluster. For example, if by chance a single bridge in b1 in Fig. 6 snaps off, the whole clusters $\mathrm{C} 1$ and $\mathrm{C} 2$ would be disconnected and could no longer be drained (assuming that there are no other sources of film connection between them and the defending fluid). Different bridges carry a different level of importance to the maintenance of the secondary network connectivity, with some (like those in b1) being responsible for the connectivity of large portions of the network, and others being essentially isolated, with no major contribution to the overall connectivity. This observation adds an interesting layer of complexity to the problem of liquid transport via film flow: in the case of usual drainage through the primary network, the basic entities of the network (pore bodies and throats) have a local role in the system's transport properties, in the sense that if a given pore body or throat is blocked, only the pores around that particular point will be affected. When film flow effects are considered, the transport happens via the secondary network of capillary bridges and liquid films and the interruption of a single entity of this network can disconnect larger regions of the system, thus playing a global role in the system's connectivity. 


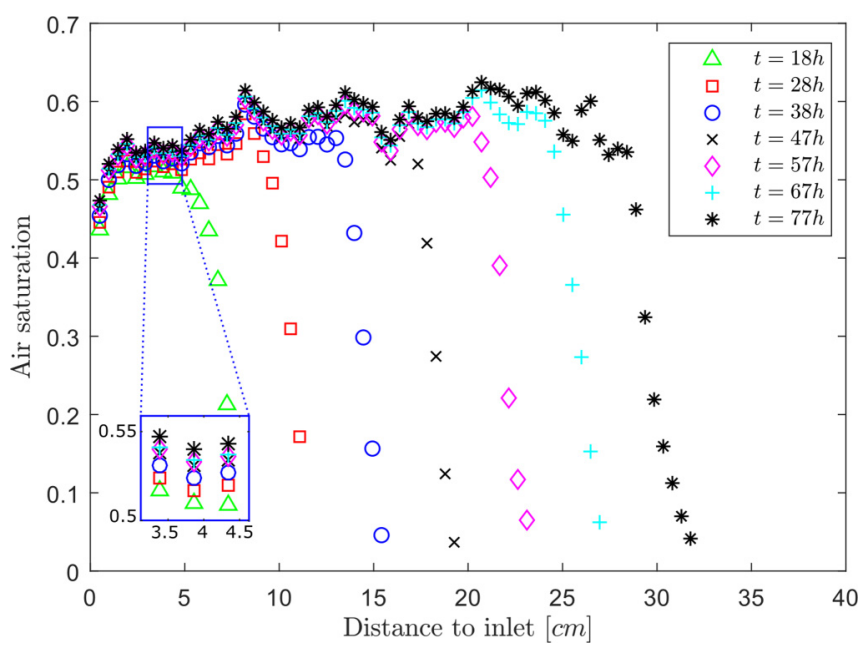

FIG. 13. Air saturation in width-spanning subwindows for an experiment with $30^{\circ}$ inclination. The subwindows are located at fixed positions with respect to the inlet for different times (shown in the legend). In the inset we can see that the increment in saturation due to late film flow effects can be very significant, amounting to almost $10 \%$ of the total saturation in the subwindow.

\section{G. Air saturation behind the front: Analysis in subwindows}

In this section we analyze the evolution of the air saturation in the porous medium using a series of width-spanning subwindows [similarly to what was done in Fig. 12(a) for the capillary bridge count]. In a system in which film flow effects are negligible, once the air-liquid interface completely passes by a region of the network, the final value of the air saturation remains constant for the whole duration of the invasion (since only pores along the interface can be drained). If film flow effects are present, this saturation can still change, since liquid clusters can still be drained for a long time due to the enhanced connectivity introduced by the capillary bridges and other possible thin liquid films, as seen in Sec. III A.

In Fig. 13, we see the spatial dependence of the air saturation along the model. Different symbols and colors denote measurements done at different times (see legend). Here we analyze the experiment with the steepest inclination $\left(30^{\circ}\right)$ and the distance is measured between the subwindow's center and the air inlet. The subwindow's height is 100 pixels $\approx 9.6 \mathrm{~mm}$ and two consecutive subwindows have a 50\% overlap. We notice that for longer times, the graph extends further to the right, since the air-liquid interface had time to invade deeper in the model. Additionally, we notice that for a given subwindow position (fixed vertical line in the graph), there is always a tendency of the saturation to increase as time progresses. When this increase happens much later than the time at which the air-liquid interface passed by the subwindow, we associate that increase to late film flow effects. These effects are represented by the "tail" events denoted in Fig. 11. In the inset in Fig. 13, we see that these late film flow effects can amount to a total increase in air saturation of the order of $10 \%$ for the subwindows closer to the inlet. We use here the term late film flow effects because from the data shown in Fig. 13 alone it is only possible to comment about the increment in saturation with respect to the initial time shown (18 $\mathrm{h}$ in the figure), and a portion of the saturation at that initial time could very well already have been due to (early) drainage via film flow. We have observed similar results for the experiments performed at lower inclination angles too, but the late film flow saturation increase was significantly lower (roughly $3 \%$ for experiments with $20^{\circ}$ inclination). This points out again the important role played by gravity in the problem. Indeed the increased power of film flow drainage for steeper angles is to be expected since gravity acts in the average direction of the flow and the defending fluid is the heavier phase. 
Finally, notice that if one simply had access to the data presented in Figs. 12(a) (capillary bridges count inside subwindows) or 13 (total air saturation inside subwindows), it would not have been possible to infer the existence of the active zone, where film flow drainage events are more likely to occur. The observation and characterization of the active zone was only possible by having access to the full dynamics of the invasion [shown in the spatiotemporal map in Fig. 5(e)].

\section{H. Two important comments about evaporation}

The process of soil evaporation bears many interesting similarities to the effects discussed here. During the so-called stage I evaporation regime [68,69] (constant rate evaporation), water is transported from the main water-air interface (lying at some depth beneath the ground level) up towards the surface where vaporization takes place. This transport happens through a set of long continuous liquid pathways. The overall process is somehow analogous to what we described here, in which liquid was transported from the trapped clusters towards the main interface via long sets of interconnected capillary bridges and intermediate liquid clusters. In the case of soil evaporation, as the transport progresses, it reaches a moment in which the continuous liquid columns start to snap off due to a reduction in its internal pressure, thus decreasing the overall connectivity of the system. The evaporation process then enters a diffusion-controlled regime (stage II), which is characterized by a reduction in evaporation rate [68]. It would be very interesting to investigate if a similar transition also occurs in the slow drainage flows described.

Still on the topic of evaporation, it is important to notice that in the experiment studied here some evaporation is also bound to take place. More importantly, due to the nature of the image analysis algorithm employed to map the film flow drainage events, pores invaded by air due to evaporation would also be mistakenly identified as film flow drainage events. We cannot remove evaporation entirely from the picture, but we have strong reasons to believe that its magnitude is not enough to cast doubt in any of the conclusions presented here. Here we present some of those reasons: (1) If evaporation effects were of considerable significance here, we would see a drift in the air saturation along the vertical direction of the model, for example in Fig. 5. Since the area closer to the inlet of the model is exposed to the air much earlier, it would have much more time to evaporate and the air saturation there would be much higher than in the middle of the model or closer to the outlet. This is not seen. In fact the drift in saturation, if any, happens to be in the opposite direction (see Fig. 13). (2) The formation of the active zone that follows the invasion front does not seem to be in line with what would be expected from evaporation. Since air is spread in the whole invaded part of the model, evaporation would lead to a widespread drainage of trapped clusters, not particularly focused in a specific region following the front. (3) We have performed a test experiment in which we have let a porous system similar to the one described here completely open to the air so that the only driving force for drainage would be evaporation (no syringe pump). We have tracked the increase in air saturation in the sample and the typical rates were much smaller than the ones observed in the experiments here. (4) The count of capillary bridges does not show any drift in the vertical direction [Fig. 12(a)]. If evaporation was too strong, we should see a reduction in the number of bridges close to the inlet, since they were exposed to the air for longer in comparison with the newer bridges closer to the outlet (measurement at breakthrough time).

\section{CONCLUSIONS}

We have performed an experimental investigation of film flow phenomena in porous media. We have shown that capillary bridges can enhance the connectivity of the network, thus making it possible for the drainage of seemingly trapped fluid regions. We have devised an algorithm to track the position of all capillary bridges and analyzed their spatial distribution along the porous network. More importantly, our experiments have shown the existence of a film flow active zone, which follows the motion of the fluid-fluid interface. This zone concentrates the majority of drainage events happening due to film flow effects. The width of the active zone seems to be reduced as the 
gravitational component along the direction of the flow increases and its mean position seems to get closer to the main invasion front.

Other kinds of secondary drainage mechanisms have been recently reported in fast drainage experiments in porous media. Hoogland et al. [70] have observed the formation of a viscous limited zone that defines the region behind the interface in which the drainage of seemingly trapped wetting phase clusters occurs, at a longer timescale than that for the main drainage. This concept is somewhat analogous to the film flow active zone observed here, in the sense that in both cases the zone delimits the region behind the interface inside which drainage of apparently trapped clusters is observed. Nevertheless, the flow regimes studied here and there are different, since the viscous forces that are held responsible for the formation of the active zone in Ref. [70], are negligible here, given that the flows that we study are slow enough to be entirely dominated by capillary forces (and gravity).

Additional studies are necessary to fully quantify the effects we have observed. In particular, longer experiments at steeper angles and larger models need to be done, in order to better justify our preliminary observations with regard to the formation and extent of the active zone. A theoretical description of the width and position of the active zone in terms of other flow and porous media parameters is still missing. The extent of interconnected regions in the porous media due to film flow can be directly influenced by the stability properties of the liquid films [71,72]. The formation and stability of capillary bridges also plays a crucial role in a number of other systems and has interestingly recently been connected to the stability properties of sandcastles [73-75].

Finally, we would like to restate the fact that since film flow phenomena can enhance the connectivity of porous networks, thus introducing new pathways for fluid transport, it is of utmost importance to consider the relative contribution of such effects when designing a simulation protocol for porous media systems. Many of the usual approaches to the problem (such as the traditional invasion percolation algorithm [39]) do not take such effects into consideration and this can lead to large errors in the estimation of quantities such as the phase saturation and other related measures that depend on it (e.g., water retention and relative permeability curves). There is a clear need for a continuation of the important efforts initiated by other researchers [46-49,51] in the direction of extending the traditional models to include film flow effects for a more accurate description of porous media flows.

\section{ACKNOWLEDGMENTS}

We gratefully acknowledge the support from the University of Oslo, University of Strasbourg, the Research Council of Norway through its Centre of Excellence funding scheme with Project No. 262644, the CNRS-INSU ALEAS program, the LIA France-Norway D-FFRACT and the EU Marie Curie ITN FLOWTRANS network.

[1] L. Furuberg, J. Feder, A. Aharony, and T. Jøssang, Dynamics of Invasion Percolation, Phys. Rev. Lett. 61, 2117 (1988).

[2] K. J. Måløy, L. Furuberg, J. Feder, and T. Jøssang, Dynamics of Slow Drainage in Porous Media, Phys. Rev. Lett. 68, 2161 (1992).

[3] R. Lenormand, Flow through porous media: Llimits of fractal pattern, Proc. R. Soc. London, Ser. A 423, 159 (1989).

[4] R. Lenormand and C. Zarcone, Capillary fingering: Percolation and fractal dimension, Transp. Porous Media 4, 599 (1989).

[5] G. Løvoll, Y. Méheust, K. J. Måløy, E. Aker, and J. Schmittbuhl, Competition of gravity, capillary and viscous forces during drainage in a two-dimensional porous medium, a pore scale study, Energy 30, 861 (2005). 
[6] D. Or, Scaling of capillary, gravity and viscous forces affecting flow morphology in unsaturated porous media, Adv. Water Resour. 31, 1129 (2008).

[7] R. Toussaint, K. J. Måløy, Y. Méheust, G. Løvoll, M. Jankov, G. Schäfer, and J. Schmittbuhl, Two-phase flow: Structure, upscaling, and consequences for macroscopic transport properties, Vadose Zone J. 11 (2012).

[8] S. Berg, H. Ott, S. A. Klapp, A. Schwing, R. Neiteler, N. Brussee, A. Makurat, L. Leu, F. Enzmann, J.-O. Schwarz, M. Kersten, S. Irvine, and M. Stampanoni, Real-time 3D imaging of Haines jumps in porous media flow, Proc. Natl. Acad. Sci. USA 110, 3755 (2013).

[9] F. Moebius and D. Or, Pore scale dynamics underlying the motion of drainage fronts in porous media, Water Resour. Res. 50, 8441 (2014).

[10] S. Schlüter, S. Berg, T. Li, H.-J. Vogel, and D. Wildenschild, Time scales of relaxation dynamics during transient conditions in two-phase flow, Water Resour. Res. 53, 4709 (2017).

[11] J. P. Stokes, D. A. Weitz, J. P. Gollub, A. Dougherty, M. O. Robbins, P. M. Chaikin, and H. M. Lindsay, Interfacial Stability of Immiscible Displacement in A Porous Medium, Phys. Rev. Lett. 57, 1718 (1986).

[12] B. Sandnes, H. A. Knudsen, K. J. Måløy, and E. G. Flekkøy, Labyrinth Patterns in Confined GranularFluid Systems, Phys. Rev. Lett. 99, 038001 (2007).

[13] H. A. Knudsen, B. Sandnes, E. G. Flekkøy, and K. J. Måløy, Granular labyrinth structures in confined geometries, Phys. Rev. E 77, 021301 (2008).

[14] B. Sandnes, E. G. Flekkøy, H. A. Knudsen, K. J. Måløy, and H. See, Patterns and flow in frictional fluid dynamics, Nat. Commun. 2, 288 (2011).

[15] F. K. Eriksen, R. Toussaint, K. J. Måløy, and E. G. Flekkøy, Invasion patterns during two-phase flow in deformable porous media, Frontiers Phys. 3, 81 (2015).

[16] G. Dumazer, B. Sandnes, M. Ayaz, K. J. Måløy, and E. G. Flekkøy, Frictional Fluid Dynamics and Plug Formation in Multiphase Millifluidic Flow, Phys. Rev. Lett. 117, 028002 (2016).

[17] J. A. Eriksen, R. Toussaint, K. J. Måløy, E. Flekkøy, O. Galland, and B. Sandnes, Pattern formation of frictional fingers in a gravitational potential, Phys. Rev. Fluids 3, 013801 (2018).

[18] L. Furuberg, K. J. Måløy, and J. Feder, Intermittent behavior in slow drainage, Phys. Rev. E 53, 966 (1996).

[19] S. Santucci, R. Planet, K. J. Måløy, and J. Ortín, Avalanches of imbibition fronts: Towards critical pinning, Europhys. Lett. 94, 46005 (2011).

[20] X. Clotet, J. Ortín, and S. Santucci, Experimental study of stable imbibition displacements in a model open fracture. I. Local avalanche dynamics, Phys. Rev. E 93, 012149 (2016).

[21] F. K. Eriksen, R. Toussaint, A. L. Turquet, K. J. Måløy, and E. G. Flekkøy, Pneumatic fractures in confined granular media, Phys. Rev. E 95, 062901 (2017).

[22] M. Moura, K. J. Måløy, and R. Toussaint, Critical behavior in porous media flow, Europhys. Lett. 118, 14004 (2017).

[23] M. Moura, K. J. Måløy, E. G. Flekkøy, and R. Toussaint, Verification of a Dynamic Scaling for the Pair Correlation Function During the Slow Drainage of A Porous Medium, Phys. Rev. Lett. 119, 154503 (2017).

[24] P. Fantinel, O. Borgman, R. Holtzman, and L. Goehring, Drying in a microfluidic chip: Experiments and simulations, Sci. Rep. 7, 15572 (2017).

[25] M. Cieplak and M. O. Robbins, Dynamical Transition in Quasistatic Fluid Invasion in Porous Media, Phys. Rev. Lett. 60, 2042 (1988).

[26] M. Cieplak and M. O. Robbins, Influence of contact angle on quasistatic fluid invasion of porous media, Phys. Rev. B 41, 11508 (1990).

[27] R. Holtzman and E. Segre, Wettability Stabilizes Fluid Invasion Into Porous Media Via Nonlocal, Cooperative Pore Filling, Phys. Rev. Lett. 115, 164501 (2015).

[28] M. Trojer, M. L. Szulczewski, and R. Juanes, Stabilizing Fluid-Fluid Displacements in Porous Media Through Wettability Alteration, Phys. Rev. Appl. 3, 054008 (2015).

[29] J. O. Helland, H. A. Friis, E. Jettestuen, and S. M. Skjæveland, Footprints of spontaneous fluid redistribution on capillary pressure in porous rock, Geophys. Res. Lett. 44, 4933 (2017). 
[30] G. Guymon, Unsaturated zone hydrology (Prentice Hall, Englewood Cliffs, NJ, 1994).

[31] S. Jellali, P. Muntzer, O. Razakarisoa, and G. Schäfer, Large scale experiment on transport of trichloroethylene in a controlled aquifer, Transp. Porous Media 44, 145 (2001).

[32] L. Xu, B. Marks, R. Toussaint, E. G. Flekkøy, and K. J. Måløy, Dispersion in fractures with ramified dissolution patterns, Frontiers Phys. 6, 29 (2018).

[33] J. M. Campbell, D. Ozturk, and B. Sandnes, Gas-Driven Fracturing of Saturated Granular Media, Phys. Rev. Appl. 8, 064029 (2017).

[34] W. A. England, A. S. Mackenzie, D. M. Mann, and T. M. Quigley, The movement and entrapment of petroleum fluids in the subsurface, J. Geol. Soc. 144, 327 (1987).

[35] M. T. Tweheyo, T. Holt, and O. Torsæter, An experimental study of the relationship between wettability and oil production characteristics, J. Pet. Sci. Eng. 24, 179 (1999).

[36] M. Shabani Afrapoli, S. Alipour, and O. Torsæter, Fundamental study of pore scale mechanisms in microbial improved oil recovery processes, Transp. Porous Media 90, 949 (2011).

[37] D. Y. C. Leung, G. Caramanna, and M. M. Maroto-Valer, An overview of current status of carbon dioxide capture and storage technologies, Renewable Sustainable Energy Rev. 39, 426 (2014).

[38] S. H. Hwang, C. Kim, H. Song, S. Son, and J. Jang, Designed architecture of multiscale porous $\mathrm{TiO}_{2}$ nanofibers for dye-sensitized solar cells photoanode, ACS Appl. Mater. Interfaces 4, 5287 (2012).

[39] D. Wilkinson and J. F. Willemsen, Invasion percolation: A new form of percolation theory, J. Phys. A: Math. Gen. 16, 3365 (1983).

[40] T. A. Witten and L. M. Sander, Diffusion-Limited Aggregation, A Kinetic Critical Phenomenon, Phys. Rev. Lett. 47, 1400 (1981).

[41] K. J. Måløy, J. Feder, and T. Jøssang, Viscous Fingering Fractals in Porous Media, Phys. Rev. Lett. 55, 2688 (1985).

[42] V. Frette, K. J. Måløy, F. Boger, J. Feder, T. Jøssang, and P. Meakin, Diffusion-limited-aggregation-like displacement structures in a three-dimensional porous medium, Phys. Rev. A 42, 3432 (1990).

[43] P. Kundu, Fluid Mechanics (Academic, Waltham, MA, 2012).

[44] C. Marle, Multiphase Flow in Porous Media (Editions Technip, Paris/Houston, 1981).

[45] B. Zhao, A. Alizadeh Pahlavan, L. Cueto-Felgueroso, and R. Juanes, Forced Wetting Transition and Bubble Pinch-Off in A Capillary Tube, Phys. Rev. Lett. 120, 084501 (2018).

[46] E. G. Flekkøy, J. Schmittbuhl, F. Løvholt, U. Oxaal, K. Jørgen Måløy, and P. Aagaard, Flow paths in wetting unsaturated flow: Experiments and simulations, Phys. Rev. E 65, 036312 (2002).

[47] R. Lenormand, C. Zarcone, and A. Sarr, Mechanisms of the displacement of one fluid by another in a network of capillary ducts, J. Fluid Mech. 135, 337 (1983).

[48] T. K. Tokunaga and J. Wan, Water film flow along fracture surfaces of porous rock, Water Resour. Res. 33, 1287 (1997).

[49] M. Goyeneche, D. Lasseux, and D. Bruneau, A film-flow model to describe free water transport during drying of a hygroscopic capillary porous medium, Transp. Porous Media 48, 125 (2002).

[50] B. Zhao, C. W. MacMinn, and R. Juanes, Wettability control on multiphase flow in patterned microfluidics, Proc. Natl. Acad. Sci. USA 113, 10251 (2016).

[51] B. K. Primkulov, S. Talman, K. Khaleghi, A. Rangriz Shokri, R. Chalaturnyk, B. Zhao, C. W. MacMinn, and R. Juanes, Quasistatic fluid-fluid displacement in porous media: Invasion-percolation through a wetting transition, Phys. Rev. Fluids 3, 104001 (2018).

[52] R. Lenormand and C. Zarcone, Role of roughness and edges during imbibition in square capillaries, Soc. Pet. Eng. 13, 1 (1984).

[53] M. Tuller, D. Or, and L. M. Dudley, Adsorption and capillary condensation in porous media: Liquid retention and interfacial configurations in angular pores, Water Resour. Res. 35, 1949 (1999).

[54] M. Tuller and D. Or, Hydraulic conductivity of variably saturated porous media: Film and corner flow in angular pore space, Water Resour. Res. 37, 1257 (2001).

[55] M. Erpelding, S. Sinha, K. T. Tallakstad, A. Hansen, E. G. Flekkøy, and K. J. Måløy, History independence of steady state in simultaneous two-phase flow through two-dimensional porous media, Phys. Rev. E 88, 053004 (2013). 
[56] M. Moura, E.-A. Fiorentino, K. J. Måløy, G. Schäfer, and R. Toussaint, Impact of sample geometry on the measurement of pressure-saturation curves: Experiments and simulations, Water Resour. Res. 51, 8900 (2015).

[57] H. S. Hele-Shaw, The flow of water, Nature (London) 58, 34 (1898).

[58] M. Jankov, G. Løvoll, H. A. Knudsen, K. J. Måløy, R. Planet, R. Toussaint, and E. G. Flekkøy, Effects of pressure oscillations on drainage in an elastic porous medium, Transp. Porous Media 84, 569 (2010).

[59] J. Bear, Dynamics of Fluids in Porous Media (Dover, New York, 2013).

[60] W. B. Haines, Studies in the physical properties of soil. V. The hysteresis effect in capillary properties, and the modes of moisture distribution associated therewith, J. Agric. Sci. 20, 97 (1930).

[61] P. Meakin, J. Feder, V. Frette, and T. Jøssang, Invasion percolation in a destabilizing gradient, Phys. Rev. A 46, 3357 (1992).

[62] Y. Méheust, G. Løvoll, K. J. Måløy, and J. Schmittbuhl, Interface scaling in a two-dimensional porous medium under combined viscous, gravity, and capillary effects, Phys. Rev. E 66, 051603 (2002).

[63] A. Birovljev, L. Furuberg, J. Feder, T. Jøssang, K. J. Måløy, and A. Aharony, Gravity Invasion Percolation in Two Dimensions: Experiment and Simulation, Phys. Rev. Lett. 67, 584 (1991).

[64] D. T. Lee and B. J. Schachter, Two algorithms for constructing a Delaunay triangulation, Int. J. Comput. Inf. Sci. 9, 219 (1980).

[65] L. Rayleigh, On the instability of jets, Proc. London Math. Soc. s1-10, 4 (1878).

[66] M. Muskat, The Flow of Homogeneous Fluids Through Porous Media (McGraw-Hill, New York, 1937).

[67] G. R. Guédon, J. D. Hyman, F. Inzoli, M. Riva, and A. Guadagnini, Influence of capillary end effects on steady-state relative permeability estimates from direct pore-scale simulations, Phys. Fluids 29, 123104 (2017).

[68] P. Lehmann, S. Assouline, and D. Or, Characteristic lengths affecting evaporative drying of porous media, Phys. Rev. E 77, 056309 (2008).

[69] D. Or, P. Lehmann, E. Shahraeeni, and N. Shokri, Advances in soil evaporation physics-A review, Vadose Zone J. 12 (2013).

[70] F. Hoogland, P. Lehmann, and D. Or, The formation of viscous limited saturation zones behind rapid drainage fronts in porous media, Water Resour. Res. 51, 9862 (2015).

[71] A. Alizadeh Pahlavan, L. Cueto-Felgueroso, A. E. Hosoi, G. H. McKinley, and R. Juanes, Thin films in partial wetting: Stability, dewetting and coarsening, J. Fluid Mech. 845, 642 (2018).

[72] G. Reiter, Dewetting of Thin Polymer Films, Phys. Rev. Lett. 68, 75 (1992).

[73] D. J. Hornbaker, R. Albert, I. Albert, A.-L. Barabási, and P. Schiffer, What keeps sandcastles standing? Nature (London) 387, 765 (1997).

[74] A. Kudrolli, Sticky sand, Nat. Mater. 7, 174 (2008).

[75] M. Pakpour, M. Habibi, P. Møller, and D. Bonn, How to construct the perfect sandcastle, Sci. Rep. 2, 549 (2012). 